\title{
Using Uniaxial Stress to Probe the Relationship between Competing Superconducting States in a Cuprate with Spin-stripe Order
}

\author{
Z. Guguchia $\odot,{ }^{1, *}$ D. Das $\odot,{ }^{1}$ C. N. Wang, ${ }^{1}$ T. Adachi $\odot,{ }^{2}$ N. Kitajima, ${ }^{3}$ M. Elender, ${ }^{1}$ F. Brückner, ${ }^{4}$ S. Ghosh,${ }^{4}$ V. Grinenko, ${ }^{4,5}$ \\ T. Shiroka $\odot,{ }^{1,6}$ M. Müllerø, ${ }^{7}$ C. Mudry, ${ }^{7,8}$ C. Baines, ${ }^{1}$ M. Bartkowiak, ${ }^{9}$ Y. Koike, ${ }^{3}$ A. Amato $\odot,{ }^{1}$ J. M. Tranquada $\odot,{ }^{10}$ \\ H.-H. Klauss, ${ }^{4}$ C. W. Hicks, ${ }^{11}$ and H. Luetkens $\oplus^{1, \dagger}$ \\ ${ }^{1}$ Laboratory for Muon Spin Spectroscopy, Paul Scherrer Institute, CH-5232 Villigen PSI, Switzerland \\ ${ }^{2}$ Department of Engineering and Applied Sciences, Sophia University, 7-1 Kioi-cho, Chiyoda-ku, Tokyo 102-8554, Japan \\ ${ }^{3}$ Department of Applied Physics, Tohoku University, 6-6-05 Aoba, Aramaki, Aoba-ku, Sendai 980-8579, Japan \\ ${ }^{4}$ Institute for Solid State and Materials Physics, Technische Universität Dresden, D-01069 Dresden, Germany \\ ${ }^{5}$ Leibniz-Institut für Festkörper- und Werkstoffforschung (IFW) Dresden, 01171 Dresden, Germany \\ ${ }^{6}$ Laboratorium für Festkörperphysik, ETH Zürich, CH-8093 Zürich, Switzerland \\ ${ }^{7}$ Condensed Matter Theory Group, Paul Scherrer Institute, CH-5232 Villigen PSI, Switzerland \\ ${ }^{8}$ Institute of Physics, École Polytechnique Fédérale de Lausanne (EPFL), CH-1015 Lausanne, Switzerland \\ ${ }^{9}$ Laboratory for Scientific Developments and Novel Materials, Paul Scherrer Institut, 5232 Villigen PSI, Switzerland \\ ${ }^{10}$ Condensed Matter Physics and Materials Science Division, Brookhaven National Laboratory, Upton, New York 11973, USA \\ ${ }^{11}$ Max Planck Institute for Chemical Physics of Solids, D-01187 Dresden, Germany
}

(Received 20 May 2020; accepted 30 July 2020; published 28 August 2020)

\begin{abstract}
We report muon spin rotation and magnetic susceptibility experiments on in-plane stress effects on the static spin-stripe order and superconductivity in the cuprate system $\mathrm{La}_{2-x} \mathrm{Ba}_{x} \mathrm{CuO}_{4}$ with $x=0.115$. An extremely low uniaxial stress of $\sim 0.1 \mathrm{GPa}$ induces a substantial decrease in the magnetic volume fraction and a dramatic rise in the onset of $3 \mathrm{D}$ superconductivity, from $\sim 10$ to $32 \mathrm{~K}$; however, the onset of at-least-2D superconductivity is much less sensitive to stress. These results show not only that largevolume-fraction spin-stripe order is anticorrelated with 3D superconducting coherence but also that these states are energetically very finely balanced. Moreover, the onset temperatures of 3D superconductivity and spin-stripe order are very similar in the large stress regime. These results strongly suggest a similar pairing mechanism for spin-stripe order and the spatially modulated 2D and uniform 3D superconducting orders, imposing an important constraint on theoretical models.
\end{abstract}

DOI: 10.1103/PhysRevLett.125.097005

Cuprate superconductors are believed to exhibit competing superconducting orders: uniform $d$ wave vs pair density wave (PDW) order [1,2]. The latter was proposed [3] to explain the observation of 2D superconductivity with depressed 3D order in $\mathrm{La}_{2-x} \mathrm{Ba}_{x} \mathrm{CuO}_{4}$ (LBCO) near $x=$ $1 / 8$ with spin-stripe order [4]. Whether these states involve distinct electron-pairing mechanisms remains unresolved.

The conventional BCS theory of superconductivity is based on the Fermi liquid model of electronic states in which uniformity in real space is assumed and electronic states are characterized entirely by their distribution in reciprocal space. Many discussions of superconducting cuprates have focused only on the nature of the bosonic "glue" responsible for electron pairing [5-7]. In contrast, others have argued that spatial inhomogeneity is intrinsic to the hole-doped cuprates and a key to understanding the pairing mechanism [8,9]. Indeed, recent many-body calculations suggest that the uniform and striped (spatially modulated) superconducting states are very close in energy $[10,11]$. At present, the mechanism that controls the competition between such states is still unclear.
Studies of LBCO can provide helpful insight into this unresolved issue since one of the most astonishing manifestations of competing ordered phases occurs in this system [12]. As shown in Fig. 1(a), the phase diagram of LBCO exhibits a large dip in the bulk 3D superconducting transition temperature, $T_{c}$, centered at $x=1 / 8$, coincident with static charge-stripe and spin-stripe orders [12] [see Fig. 1(b)]. Nevertheless, 2D superconductivity onsets at $40 \mathrm{~K}$, together with spin-stripe order [4]. A finite interlayer Josephson coupling would normally be expected to lock the phases of the superconducting wave function between the layers, resulting in 3D order. To explain the apparent frustration of interlayer Josephson coupling, pair-densitywave order within the layers has been proposed $[3,13]$, which is compatible with both the charge-stripe and spinstripe orders.

What happens when the stripe order is perturbed? A recent transport study on LBCO $x=0.125$ under strong magnetic fields (applied along the $c$ axis) provided evidence that the putative pairing within the charge stripes is remarkably robust [14]. High pressure experiments on 


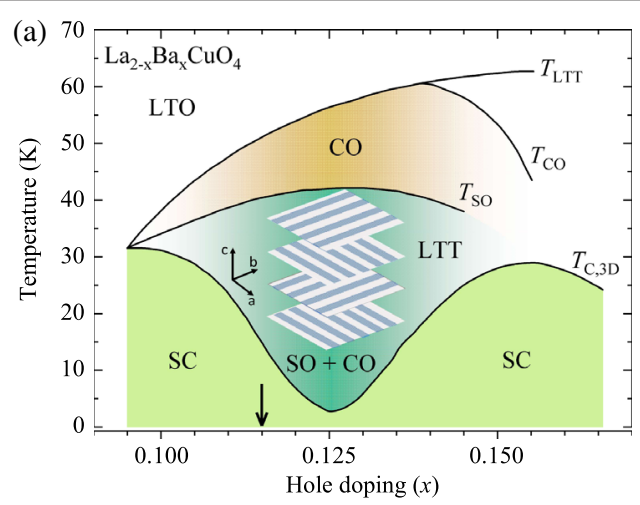

(b)

(c)
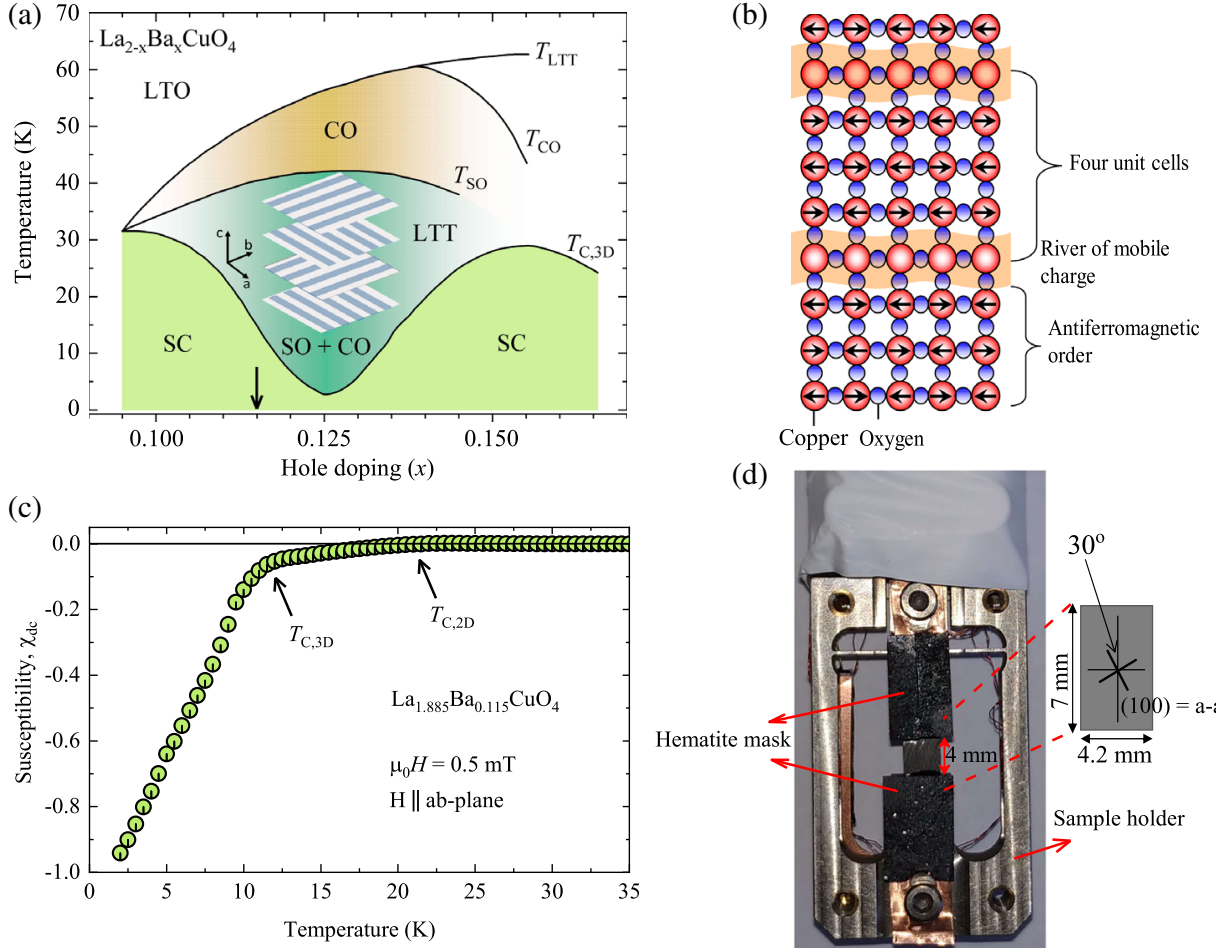

(d)

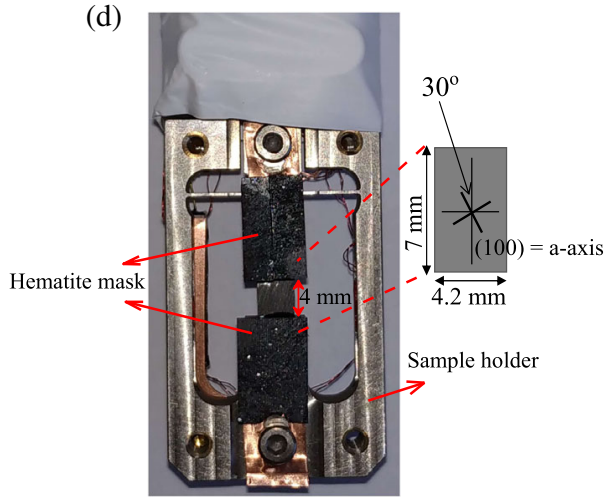

FIG. 1. (a) The schematic temperature-doping phase diagram of $\mathrm{La}_{2-x} \mathrm{Ba}_{x} \mathrm{CuO}_{4}$. The arrow indicates the present doping value. The inset illustrates the orthogonal stripe directions between neighboring layers. The various phases in the phase diagram are denoted as follows: charge-stripe order (CO), low-temperature orthorhombic (LTO), low-temperature tetragonal (LTT), spin-stripe order (SO), and 3D superconductivity (SC). (b) Illustration of a domain of spin-stripe and charge-stripe orders for a layer of LBCO, indicating the periods of the charge (4a) and spin (8a) modulations. (c) The temperature dependence of the zero-field-cooled magnetic susceptibility for $\mathrm{La}_{1.885} \mathrm{Ba}_{0.115} \mathrm{CuO}_{4}$. (d) The uniaxial stress sample holder used for the $\mu \mathrm{SR}$ experiments.

LBCO $x=0.125$ have found that the impact on the 3D superconducting transition temperature is quite modest, even beyond the critical pressure where the long-range structural anisotropy, assumed necessary to pin the charge stripes, is absent [15,16]. An optical pump-probe study of LBCO $x=0.115$ found evidence for the suppression of charge-stripe order together with enhanced interlayer superconducting coherence [17]; however, the dynamic character of such measurements is not without ambiguity.

Here we perturb a crystal of LBCO $x=0.115$ [18] with inplane compressive stress applied to the $\mathrm{CuO}_{2}$ layers, using an in situ piezoelectrically driven stress device [19-21], while microscopically probing the spin-stripe order with muon spin rotation $(\mu \mathrm{SR})$ [16,22-26] spectroscopy and the superconducting transitions with ac susceptibility. The details on the $\mu \mathrm{SR}$ technique, data analysis, the uniaxial stress device, and the sample mounting are given in the Supplemental Material [27].

The diamagnetic response of the LBCO $x=0.115$ crystal, measured before mounting in the stress apparatus, is shown in Fig. 1(c). The sample was zero-field (ZF) cooled and then measured in a dc field of $\mu_{0} H=0.5 \mathrm{mT}$. The field was applied parallel to the $\mathrm{CuO}_{2}$ planes so that the resulting shielding currents must flow between the layers, making the measurement sensitive to the onset of $3 \mathrm{D}$ superconductivity near $11 \mathrm{~K}$, consistent with previous work $[28,29]$. The onset of weak diamagnetism near $22 \mathrm{~K}$ corresponds to the 2D superconducting order, as confirmed by the $T$ dependence of the in-plane resistivity [Fig. 2(b)], which effectively drops to zero at $22 \mathrm{~K}$. Besides the SC transition, an anomaly is seen in the resistivity data at $T_{\mathrm{LTT}}$ $50 \mathrm{~K}$ [Fig. 2(b)], which is related to the structural phase transition from a high temperature orthogonal (LTO) to a low temperature tetragonal (LTT) phase.

A photograph of the $\mu$ SR sample holder, which is used to apply uniaxial stress to the LBCO-0.115 sample, is shown in Fig. 1(d). The compressive stress was applied at an angle of $30^{\circ}$ to the $\mathrm{Cu}-\mathrm{O}$ bond direction, denoted as [100]. A previous study of $\mathrm{La}_{1.64} \mathrm{Eu}_{0.2} \mathrm{Sr}_{0.16} \mathrm{CuO}_{4}$ found a rapid enhancement of bulk $T_{c}$ under in-plane uniaxial stress, especially for stress along [110] directions [30]. To monitor the effect of stress on superconductivity in our case, in situ ac susceptibility measurements were performed, with an excitation field mostly along the $c$ axis, either just before or after the $\mu \mathrm{SR}$ measurements, at each stress value. The results are shown in Fig. 2(a). A comparison with the dc measurement reveals that some stress is present even when the voltage applied to the piezoelectric force generator is zero, possibly due to differential thermal contraction (see Supplemental Material [27] for the details of the device). 

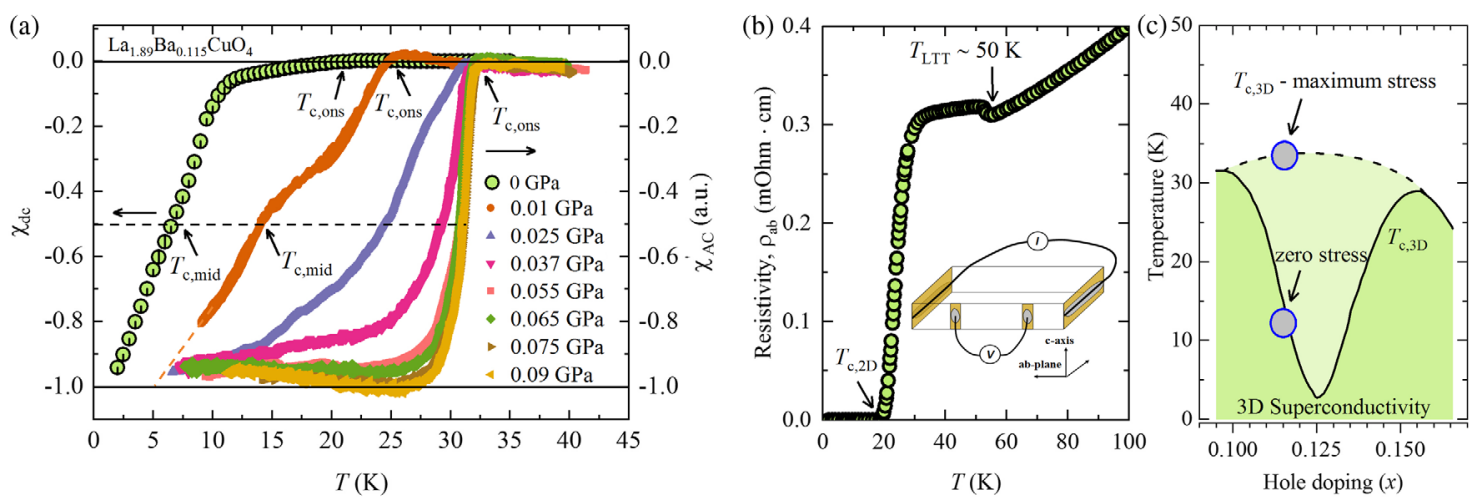

FIG. 2. (a) The temperature dependence of the (dia)magnetic susceptibility for $\mathrm{La}_{1.885} \mathrm{Ba}_{0.115} \mathrm{CuO}_{4}$ recorded at ambient (left axis) and under various degrees of compressive stress (right axis). Arrows mark the onset temperature $T_{c, \text { ons }}$ and the temperature $T_{c, \text { mid }}$ at which $\chi_{\mathrm{dc}}=-0.5$. (b) The temperature dependence of the in-plane resistivity (without stress). Electrodes and contacts were placed on the sample as schematically shown in the inset. (c) Schematic temperature-doping phase diagram, indicating the enhancement of 3D SC critical temperature $T_{c, 3 \mathrm{D}}$ under stress for the $\mathrm{LBCO} x=0.115$ sample. The value of the $T_{c, 3 \mathrm{D}}$ under maximum stress is quite similar to the optimal value of SC critical temperature observed in LBCO. The dashed line represents the hypothetical SC phase boundary expected under applied stress in the broader region around $1 / 8$ doping.

To characterize the changes in superconducting critical temperature, we identify the onset temperature $T_{c \text {,ons }}$ (which equals $T_{c, 2 \mathrm{D}}$ at zero stress) and midpoint temperature $T_{c \text {,mid }}$ (which is a good measure of 3D SC order temperature $T_{c, 3 \mathrm{D}}$ ), as indicated in Fig. 2(a), and take the strongest diamagnetic response seen to indicate $100 \%$ volume-fraction superconductivity. As one can see, the compressive stress causes a rapid linear rise of $T_{c \text {,mid }}$ from 7 to $32 \mathrm{~K}$ (with a growth rate of $62.5 \mathrm{~K} / \mathrm{kbar}$ ), where it saturates. The change in $T_{c \text {,ons }}$ is much more modest. Namely, $T_{c \text {,ons }}$ increases from 22 to $32 \mathrm{~K}$. Consequently, as indicated in Fig. 2(c), the bulk transition $T_{c, 3 \mathrm{D}}$ rises from a very suppressed value to the one that is quite similar to the optimal value of SC critical temperature observed in LBCO or $\mathrm{La}_{2-x} \mathrm{Sr}_{x} \mathrm{CuO}_{4}$ (LSCO) at the same doping level [31].

The evolution of the spin-stripe order with compressive stress was characterized by a combination of weak transverse-field (TF) and $\mathrm{ZF} \mu \mathrm{SR}$ measurements. In a $\mu \mathrm{SR}$ experiment, positive muons are implanted into the sample, where each muon spin precesses in the local magnetic field. The time dependent polarization $P(t)$ of the ensemble is monitored by detecting the positrons ejected when the muons decay (see the Methods section in the Supplemental Material [27] for details). $\mu \mathrm{SR}$ is an ideal technique for probing materials such as cuprates, where competing phases may exist together and form microscopic inhomogeneity. Measuring the asymmetry between muons counted in detectors on opposite sides of the sample and then dividing by the maximum possible signal, one obtains the muon polarization function $P_{\mathrm{TF}}(t)$, several examples of which are shown in Fig. 3(a). In a weak-TF measurement, muons in regions that have no local magnetic order precess in the small applied field. Muons that stop in regions with magnetic order, and therefore experience the vector sum of external and internal fields, dephase rapidly. This causes a rapid reduction in the observable $P_{\mathrm{TF}}(0)$ (see the Methods
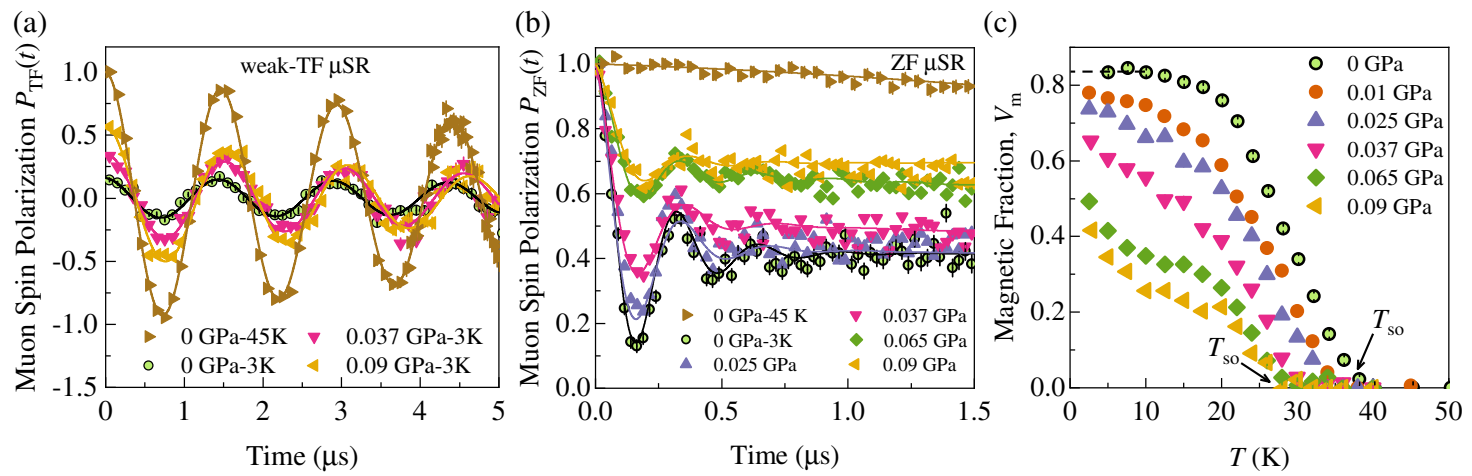

FIG. 3. (a) The weak-TF $\mu \mathrm{SR}$ spectra recorded for $\mathrm{La}_{1.885} \mathrm{Ba}_{0.115} \mathrm{CuO}_{4}$ at the base temperature $T=3 \mathrm{~K}$ under various degrees of compressive stress. (b) The zero-field $\mu \mathrm{SR}$ spectra recorded at the base temperature under various stresses. (c) The temperature dependence of the magnetically ordered volume fraction recorded under various stresses, as deduced from the TF $\mu$ SR data shown in panel (a). 


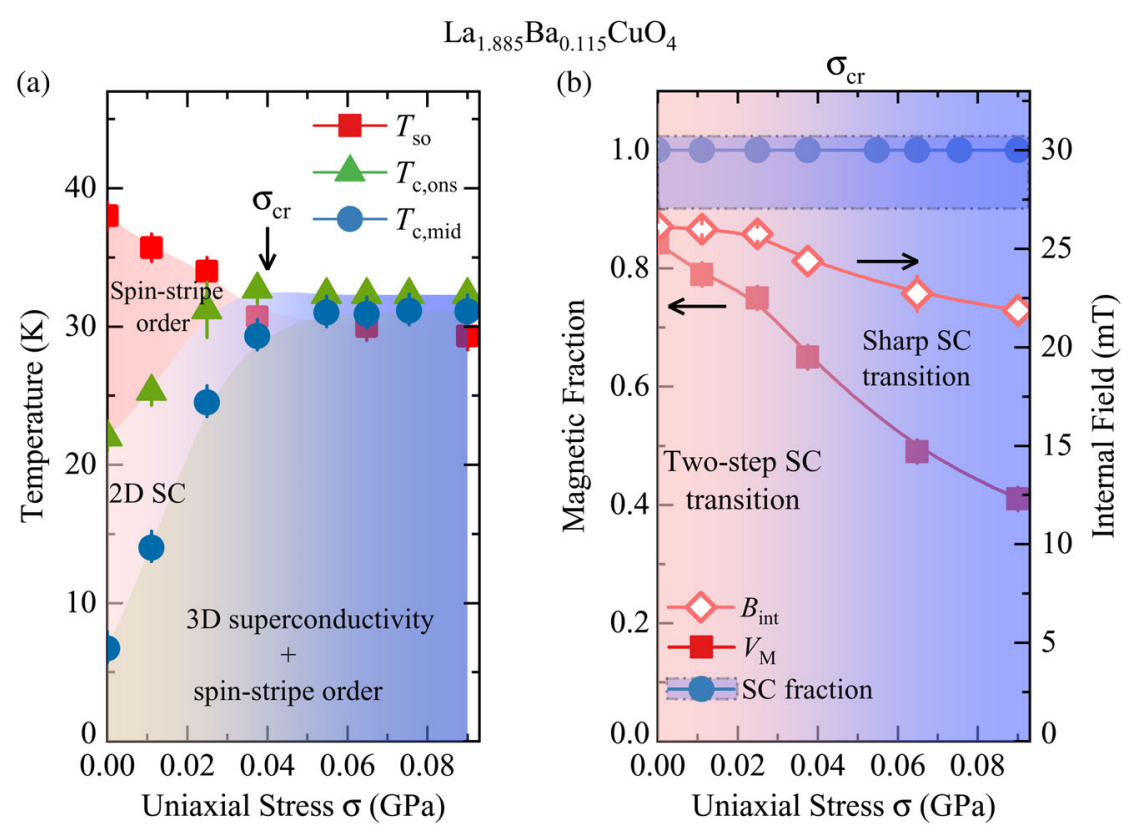

FIG. 4. (a) The compressive stress dependence of the SC transition temperatures and of static spin-stripe order temperature $T_{\text {so }}$ in LBCO $x=0.115$. Black arrow marks the critical stress value $\sigma_{\mathrm{cr}}$, above which a sharp 3D SC transition is established. (b) The stress dependence of the base- $T$ value $(T=3 \mathrm{~K})$ of the magnetically ordered fraction $V_{m}$ and the value of the internal magnetic field $B_{\text {int }}$. The SC fraction is only schematic.

section in the Supplemental Material [27]). Thus, the maximum amplitude of the weak- $\mathrm{TF} \mu \mathrm{SR}$ signal is proportional to the nonmagnetic fraction, and the magnetic volume fraction $V_{m}$ can be taken to be $1-P_{\mathrm{TF}}(0)$. At $45 \mathrm{~K}$ and zero applied stress, $P_{\mathrm{TF}}(0)=1$, indicating that there is no magnetic order. At $3 \mathrm{~K}, P_{\mathrm{TF}}(0)$ is greatly reduced, indicating the development of magnetic order in most of the sample volume. Plots of the temperature dependence of $V_{m}$ for various stresses are presented in Fig. 3(c). As stress is applied, there is a decrease in the spinordering temperature $T_{\mathrm{so}}$ from $\sim 38 \mathrm{~K}$ at $0 \mathrm{GPa}$ to $\sim 30 \mathrm{~K}$ at $0.09 \mathrm{GPa}$. $V_{m}$ decreases much more steeply: at $3 \mathrm{~K}$ by a factor of 2 and at $10 \mathrm{~K}$ by factor of 3 at $0.09 \mathrm{GPa}$.

In $\mathrm{ZF} \mu \mathrm{SR}$ measurements, the muon spins precess exclusively in the internal local field associated with the static magnetic order, with the collective response averaging over the distribution of muon sites relative to the local modulations of the internal field. As shown in Fig. 3(b), several oscillations remain clearly observable under increasing compressive stress values despite a strong reduction in magnetic volume fraction. The characteristic internal field $B_{\text {int }}$ at the muon stopping site can be extracted from the oscillation frequency, as described in the Methods section in the Supplemental Material [27].

Our overall results are summarized in Fig. 4. The spinstripe order temperature $T_{\mathrm{so}}$ and superconducting transition temperatures are plotted against stress in Fig. 4(a). The stress dependence of the magnetic volume fraction and internal magnetic field at $3 \mathrm{~K}$ are shown in Fig. 4(b). Figure 4(a) shows that the crossover from $2 \mathrm{D}$ to $3 \mathrm{D}$ superconducting order occurs at a characteristic uniaxial stress of $\sigma_{\mathrm{cr}}=0.04 \mathrm{GPa}$. The dominant change of the spinstripe order induced by uniaxial stress is a strong reduction in $V_{m} . V_{m}$ starts to decrease more rapidly above $\sigma_{\mathrm{cr}}$, and the reduced $V_{m}$ correlates with the increase (and saturation) of $T_{c \text {,mid }}$. The 2D-3D crossover has the appearance of a transition that is intrinsically first order but broadened by stress inhomogeneity. Further experiments under extremely homogeneous stress conditions are needed to shed light on the precise nature of stress-induced 2D-3D transition. We note that only a modest stress-induced decrease in $T_{\text {so }}$ [Fig. 4(a)] and in $B_{\text {int }}$ [Fig. 4(b)] is resolved, indicating that the magnetic structure is well ordered also under stress. Interestingly, $T_{\text {so }}$ decreases to essentially match $T_{c \text {,mid }}$ for $\sigma>\sigma_{\mathrm{cr}}$. There might be a several reasons for the decrease of $B_{\text {int }}$ [Fig. 4(b)]: (1) a decrease of the ordered magnetic moment, (2) a slight shift of the muon position due to the modification of the crystal structure, or (3) a continuous reorientation of the spin-stripe structure (see Supplemental Material [27]) due to a possibly weakened local pinning to the atomic structure as a result of the applied stress.

To interpret these results, we first recall that the prevalent electronic structure far away from $x=1 / 8$ is a spatially uniform state, with neither magnetic nor charge order but with uniform $d$-wave superconductivity. Close to $x=1 / 8$ a competing phase emerges with charge and spin stripes pinned along the $a$ and $b$ axes [12,32], their orientation alternating from layer to layer [33] [see inset in Fig. 1(a)]. The difference in ordering temperatures for $2 \mathrm{D}$ and $3 \mathrm{D}$ 
superconducting order in LBCO with $x$ near $1 / 8$, as we observe here, implies a strong frustration of the interlayer Josephson coupling. This strong frustration has been rationalized by suggesting PDW order in the layers, with the sign of the superconducting order parameter alternating from stripe to stripe such that the Josephson coupling between adjacent layers with orthogonal stripes is perfectly geometrically frustrated $[2,3]$. Further experimental support for PDW order is provided by recent STM data [34]. A perfect stripe phase would, however, suppress the 3D ordering temperature much more than what is observed for LBCO-0.115. This indicates that perfect frustration is probably lifted, either by local deviations from perfect orthogonality of the stripes in adjacent layers or by the inclusion of patches that remain in the uniform phase. The off-stoichiometric doping in LBCO-0.115 means that local inhomogeneity is likely to be stronger and patches of uniform superconductivity are likely to be able to establish percolative 3D phase coherence at a higher temperature than at $x=1 / 8$, and indeed, at zero stress $V_{m}$ is $85 \%$, not $100 \%$, showing that the electronic structure of the sample is not homogeneous.

Applied stress can reinforce both types of deviations from perfect geometric frustration. Since stress distorts the crystal from its tetragonal symmetry, it disfavors orthogonal stripes and thus is expected to promote the abundance of uniform patches. Patches in adjacent layers whose projections overlap mediate a nonzero interlayer coupling. However, as long as the patches are sparse, the PDW of the stripes dominates the intralayer physics, and the intralayer order parameter has a vanishing uniform component. Accordingly, the interlayer couplings remain frustrated, very much like in an XY spin glass. These couplings can nevertheless induce an amorphous (glass-like) superconducting $3 \mathrm{D}$ order at a finite temperature $T_{c, 3 \mathrm{D}}$, which in general is lower than $T_{c, 2 \mathrm{D}}$. As the fraction of uniform patches increases, $T_{c, 3 \mathrm{D}}$ grows. Beyond a critical fraction of such patches, the superconducting phase will develop a uniform $(Q=0)$ long-range order both within and between the planes. At that point, $T_{c, 3 \mathrm{D}}$ must coincide with $T_{c, 2 \mathrm{D}}$.

Since spatially uniform $d$-wave superconducting order in cuprates is empirically known not to show internal static magnetic order, the scenario of a stress enhanced abundance of uniform patches is consistent with our observation of a significant decrease in magnetic volume fraction that correlates with the increase of $T_{c, 3 \mathrm{D}}$. A mere reorientation of stripes would instead be hard to reconcile with a decrease in $V_{m}$. Given the drastic change in the superconducting order, it seems likely that the stress reduces the LTT tilting angle $[35,36]$ or induces a transition to the LTO phase in some parts of the sample like the one present in the superconducting phase of LSCO [32], where 3D superconductivity with a similar $T_{c}$ has been observed to coexist with $V_{m} \approx 20 \%$ [37]. The observation of nonlinear stressstrain (force-displacement) response (cf. Supplemental
Material [27]) provides indirect evidence for structural transitions that could lead to the formation of additional uniform patches. In this context, it is worth pointing out a recent theoretical work on the coexistence of zero and finite momentum superconductivity [38] in which a first order transition between a state with leading PDW order and subleading uniform $\mathrm{SC}$ order and a state where the roles are reversed follows naturally in a model with local attraction and repulsive pair hopping.

A key point here is that the variation in onset temperatures of superconductivity as stress shifts the balance from $2 \mathrm{D}$ to $3 \mathrm{D}$ superconducting order is quite modest. This suggests that the underlying (local) pairing mechanisms are essentially the same in the alternative superconducting states with and without spin-stripe order. What evolves instead is the degree to which fluctuations play a role and the way the bulk coherence is established. Remarkably, the stress required to establish the 3D coherence is very small: $\sigma_{\text {cr }} \sim 0.04 \mathrm{GPa}$ (strain of $\sim 0.05 \%$ ), which is much smaller than the stress $\sim 1 \mathrm{GPa}$ (strain of $1 \%$ ) that is required to, for instance, induce 3D charge density wave order in $\sim 1 / 8$ doped yttrium barium copper oxide (YBCO) [39]. Such tiny stress values are not expected to drive strong changes in the underlying electronic structure in materials such as LBCO. Thus, we conclude that the PDW state in unstressed LBCO-0.115 and the 3D superconductivity in uniaxially stressed LBCO are very close in free energy. Moreover, the onset temperatures for the 3D superconductivity and spinstripe order are quite similar in the not-so-frustrated large stress regime (beyond the critical stress $\sigma_{\mathrm{cr}} \sim 0.04 \mathrm{GPa}$ ), from which we infer that the same kind of electronic interactions are responsible for both phenomena. Given that photoemission studies on LBCO and LSCO at compositions with spin-stripe order indicate the absence of sharply defined quasiparticle peaks [40,41], it appears that any realistic theory of the pairing should not rely on Fermiliquid theory as a starting point.

Our experiment has important implications for the field of high-temperature superconductivity and, hence, should stimulate the development of an adequate theory. It also leads to new questions such as what is the impact of the stress on the crystal structure and charge-stripe order? How do these effects vary with doping? How does the transition between PDW and uniform $d$-wave SC states happen? Future experiments will be needed to provide answers. In any case, our results provide a new example of the intriguing behavior that can be uncovered by studies with applied uniaxial stress.

In conclusion, we use muon spin rotation and magnetic susceptibility measurements to follow the evolution of spin-stripe order and superconductivity in LBCO with $x=0.115$ as a function of stress applied within the $\mathrm{CuO}_{2}$ planes. We observed that an extremely low uniaxial stress of $\sim 0.1 \mathrm{GPa}$ causes a substantial reduction of the magnetic volume fraction and a dramatic rise from 
$\sim 10$ to $32 \mathrm{~K}$ in the onset of 3D superconductivity, while the onset of 2D superconducting order weakly and continuously shifts to the one of the 3D order. Moreover, the onset temperatures for 3D superconductivity and spin-stripe order are quite similar in the large stress regime. These results suggest that the underlying pairing mechanisms are essentially the same in the spatially modulated 2D and the uniform 3D superconducting states and that the presence of large-volume-fraction spin-stripe order locally inhibits the development of 3D superconductivity.

This work is based on experiments performed at the Swiss Muon Source $\mathrm{S} \mu \mathrm{S}$, Paul Scherrer Institute, Villigen, Switzerland. J. M. T. was supported at Brookhaven by the U.S. Department of Energy (DOE), Office of Basic Energy Sciences, Division of Materials Sciences and Engineering, under Contract No. DE-SC0012704. This work has been also supported by the Deutsche Forschungsgemeinschaft (GR 4667/1, GRK 1621, and SFB 1143). T. A. was supported by JSPS KAKENHI through Grant No. JP19H01841. We are grateful to S. A. Kivelson for helpful comments. M. M. was supported by the SNF through Grant No. 200021_166271.

Z. G., D. D., and C.N.W. contributed equally to the experiments.

*Corresponding author.

zurab.guguchia@psi.ch

${ }^{\dagger}$ Corresponding author.

hubertus.luetkens@psi.ch

[1] N. J. Robinson, P. D. Johnson, T. M. Rice, and A. M. Tsvelik, Anomalies in the pseudogap phase of the cuprates: Competing ground states and the role of umklapp scattering, Rep. Prog. Phys. 82, 126501 (2019).

[2] D. F. Agterberg, J. C. S. Davis, S. D. Edkins, E. Fradkin, D. J. V. Harlingen, S. A. Kivelson, L. Radzihovsky, J. M. Tranquada, and Y. Wang, The physics of pair density waves, Annu. Rev. Condens. Matter Phys. 11, 231 (2020).

[3] E. Berg, E. Fradkin, E.-A. Kim, S. A. Kivelson, V. Oganesyan, J. M. Tranquada, and S. C. Zhang, Dynamical Layer Decoupling in a Stripe-Ordered High- $T_{c}$ Superconductor, Phys. Rev. Lett. 99, 127003 (2007).

[4] Q. Li, M. Hücker, G. D. Gu, A. M. Tsvelik, and J. M. Tranquada, Two-Dimensional Superconducting Fluctuations in Stripe-Ordered $\mathrm{La}_{1.875} \mathrm{Ba}_{0.125} \mathrm{CuO}_{4}$, Phys. Rev. Lett. 99, 067001 (2007).

[5] A. Abanov, A. V. Chubukov, and J. Schmalian, Fingerprints of spin mediated pairing in cuprates, J. Electron Spectrosc. Relat. Phenom. 117-118, 129 (2001).

[6] I. Vekhter and C. M. Varma, Proposal to Determine the Spectrum of Pairing Glue in High-Temperature Superconductors, Phys. Rev. Lett. 90, 237003 (2003).

[7] T. Dahm, V. Hinkov, S. V. Borisenko, A. A. Kordyuk, V. B. Zabolotnyy, J. Fink, B. Büchner, D. J. Scalapino, W. Hanke, and B. Keimer, Strength of the spin-fluctuation-mediated pairing interaction in a high-temperature superconductor, Nat. Phys. 5, 217 (2009).
[8] V. J. Emery, S. A. Kivelson, and J. M. Tranquada, Stripe phases in high-temperature superconductors, Proc. Natl. Acad. Sci. U.S.A. 96, 8814 (1999).

[9] E. Fradkin, S. A. Kivelson, and J. M. Tranquada, Colloquium: Theory of intertwined orders in high temperature superconductors, Rev. Mod. Phys. 87, 457 (2015).

[10] P. Corboz, T. M. Rice, and M. Troyer, Competing States in the $t$ - $J$ Model: Uniform $d$-Wave State versus Stripe State, Phys. Rev. Lett. 113, 046402 (2014).

[11] B.-X. Zheng, C.-M. Chung, P. Corboz, G. Ehlers, M.-P. Qin, R. M. Noack, H. Shi, S. R. White, S. Zhang, and G. K.-L. Chan, Stripe order in the underdoped region of the two-dimensional Hubbard model, Science 358, 1155 (2017).

[12] M. Hücker, M. v. Zimmermann, G. D. Gu, Z. J. Xu, J. S. Wen, G. Xu, H. J. Kang, A. Zheludev, and J.M. Tranquada, Stripe order in superconducting $\mathrm{La}_{2-x} \mathrm{Ba}_{x} \mathrm{CuO}_{4}$ $(0.095 \leq x \leq 0.155)$, Phys. Rev. B 83, 104506 (2011).

[13] A. Himeda, T. Kato, and M. Ogata, Stripe States with Spatially Oscillating $d$-Wave Superconductivity in the TwoDimensional $t-t^{\prime}-J$ Model, Phys. Rev. Lett. 88, 117001 (2002).

[14] Y. Li, J. Terzic, P. G. Baity, D. Popović, G. D. Gu, Q. Li, A. M. Tsvelik, and J.M. Tranquada, Tuning from failed superconductor to failed insulator with magnetic field, Sci. Adv. 5, eaav7686 (2019).

[15] M. Hücker, M. v. Zimmermann, M. Debessai, J. S. Schilling, J. M. Tranquada, and G. D. Gu, Spontaneous Symmetry Breaking by Charge Stripes in the High Pressure Phase of Superconducting $\mathrm{La}_{1.875} \mathrm{Ba}_{0.125} \mathrm{CuO}_{4}$, Phys. Rev. Lett. 104, 057004 (2010).

[16] Z. Guguchia, A. Maisuradze, G. Ghambashidze, R. Khasanov, A. Shengelaya, and H. Keller, Tuning the static spin-stripe phase and superconductivity in $\mathrm{La}_{2-x} \mathrm{Ba}_{x} \mathrm{CuO}_{4}$ $(x=1 / 8)$ by hydrostatic pressure, New J. Phys. 15, 093005 (2013).

[17] V. Khanna, R. Mankowsky, M. Petrich, H. Bromberger, S. A. Cavill, E. Möhr-Vorobeva, D. Nicoletti, Y. Laplace, G. D. Gu, J. P. Hill, M. Först, A. Cavalleri, and S. S. Dhesi, Restoring interlayer Josephson coupling in $\mathrm{La}_{1.885} \mathrm{Ba}_{0.115} \mathrm{CuO}_{4}$ by charge transfer melting of stripe order, Phys. Rev. B 93, 224522 (2016).

[18] T. Adachi, T. Noji, and Y. Koike, Crystal growth, transport properties, and crystal structure of the single-crystal $\mathrm{La}_{2-x} \mathrm{Ba}_{x} \mathrm{CuO}_{4} \quad(\mathrm{x}=0.11)$, Phys. Rev. B 64, 144524 (2001).

[19] C. W. Hicks, S. Ghosh, M. E. Barber, and H.-H. Klauss, Piezoelectric-driven uniaxial stress apparatus for muon spin rotation, J. Phys. Soc. Jpn. Conf. Proc. 21, 011040 (2018).

[20] V. Grinenko et al., Split superconducting and time-reversal symmetry-breaking transitions, and magnetic order in $\mathrm{Sr}_{2} \mathrm{RuO}_{4}$ under uniaxial stress, arXiv:2001.08152.

[21] S. Ghosh et al., (to be published).

[22] G. M. Luke, L. P. Le, B. J. Sternlieb, W. D. Wu, Y. J. Uemura, J. H. Brewer, T. M. Riseman, S. Ishibashi, and S. Uchida, Static magnetic order in $\mathrm{La}_{1.875} \mathrm{Ba}_{0.125} \mathrm{CuO}_{4}$, Physica (Amsterdam) 185-9C, 1175 (1991).

[23] H. H. Klauss, Spin stripe order and superconductivity in layered transition metal oxides, J. Phys. Condens. Matter 16, S4457 (2004). 
[24] A. Suter and B. Wojek, musrfit: A free platformindependent framework for $\mu \mathrm{SR}$ data analysis, Phys. Procedia 30, 69 (2012).

[25] H. E. Mohottala, B. O. Wells, J. I. Budnick, W. A. Hines, C. Niedermayer, L. Udby, C. Bernhard, A. R. Moodenbaugh, and F.-C. Chou, Phase separation in superoxygenated $\mathrm{La}_{2-x} \mathrm{Sr}_{x} \mathrm{CuO}_{4+y}$, Nat. Mater. 5, 377 (2006).

[26] P. Dalmas de Reotier and A. Yaouanc, Muon spin rotation in magnetic materials, J. Phys. Condens. Matter 9, 9113 (1997).

[27] See Supplemental Material at http://link.aps.org/supplemental/ 10.1103/PhysRevLett.125.097005 for the details of sample preparation, $\mu \mathrm{SR}$ experiment, technical details of the uniaxial stress device and a brief qualitative discussion on the results presented in the main paper.

[28] J. M. Tranquada, G. D. Gu, M. Hücker, Q. Jie, H.-J. Kang, R. Klingeler, Q. Li, N. Tristan, J. S. Wen, G. Y. $\mathrm{Xu}, \mathrm{Z}$. J. Xu, J. Zhou, and M. v. Zimmermann, Evidence for unusual superconducting correlations coexisting with stripe order in $\mathrm{La}_{1.875} \mathrm{Ba}_{0.125} \mathrm{CuO}_{4}$, Phys. Rev. B 78, 174529 (2008).

[29] Z. Guguchia, R. Khasanov, A. Shengelaya, E. Pomjakushina, S. J. L. Billinge, A. Amato, E. Morenzoni, and H. Keller, Cooperative coupling of static magnetism and bulk superconductivity in the stripe phase of $\mathrm{La}_{2-x} \mathrm{Ba}_{x} \mathrm{CuO}_{4}$ : Pressure- and doping-dependent studies, Phys. Rev. B 94, 214511 (2016).

[30] N. Takeshita, T. Sasagawa, T. Sugioka, Y. Tokura, and H. Takagi, Gigantic anisotropic uniaxial pressure effect on superconductivity within the $\mathrm{CuO}_{2}$ plane of $\mathrm{La}_{1.64} \mathrm{Eu}_{0.2} \mathrm{Sr}_{0.16} \mathrm{CuO}_{4}$ : Strain control of stripe criticality, J. Phys. Soc. Jpn. 73, 1123 (2004).

[31] H. Takagi, T. Ido, S. Ishibashi, M. Uota, S. Uchida, and Y. Tokura, Superconductor-to-nonsuperconductor transition in $\left(\mathrm{La}_{1-\mathrm{x}} \mathrm{Sr}_{\mathrm{x}}\right)_{2} \mathrm{CuO}_{4}$ as investigated by transport and magnetic measurements, Phys. Rev. B 40, 2254 (1989).

[32] J. D. Axe and M. K. Crawford, Structural instabilities in lanthanum cuprate superconductors, J. Low Temp. Phys. 95, 271 (1994).

[33] M. v. Zimmermann, A. Vigliante, T. Niemöller, N. Ichikawa, T. Frello, J. Madsen, P. Wochner, S. Uchida, N. H. Andersen, J. M. Tranquada, D. Gibbs, and
J. R. Schneider, Hard-X-ray diffraction study of charge stripe order in $\mathrm{La}_{1.48} \mathrm{Nd}_{0.4} \mathrm{Sr}_{0.12} \mathrm{CuO}_{4}$, Europhys. Lett. 41, 629 (1998).

[34] Z. Du, H. Li, S. H. Joo, E. P. Donoway, J. Lee, J. C. Séamus Davis, G. Gu, P. D. Johnson, and K. Fujita, Imaging the energy gap modulations of the cuprate pair-density-wave state, Nature (London) 580, 65 (2020).

[35] B. Nachumi, Y. Fudamoto, A. Keren, K. M. Kojima, M. Larkin, G. M. Luke, J. Merrin, O. Tchernyshyov, Y. J. Uemura, N. Ichikawa, M. Goto, H. Takagi, S. Uchida, M. K. Crawford, E. M. McCarron, D. E. MacLaughlin, and R. H. Heffner, Muon spin relaxation study of the stripe phase order in $\mathrm{La}_{1.6-x} \mathrm{Nd}_{0.4} \mathrm{Sr}_{x} \mathrm{CuO}_{4}$ and related 214 cuprates, Phys. Rev. B 58, 8760 (1998).

[36] H.-H. Klauss, W. Wagener, M. Hillberg, W. Kopmann, H. Walf, F. J. Litterst, M. Hücker, and B. Büchner, From Antiferromagnetic Order to Static Magnetic Stripes: The Phase Diagram of $(\mathrm{La}, \mathrm{Eu})_{2-x} \mathrm{Sr}_{x} \mathrm{CuO}_{4}$, Phys. Rev. Lett. 85, 4590 (2000).

[37] A. T. Savici, Y. Fudamoto, I. M. Gat, T. Ito, M. I. Larkin, Y. J. Uemura, G. M. Luke, K. M. Kojima, Y. S. Lee, M. A. Kastner, R. J. Birgeneau, and K. Yamada, Muon spin relaxation studies of incommensurate magnetism and superconductivity in stage- $4 \mathrm{La}_{2} \mathrm{CuO}_{4.11}$ and $\mathrm{La}_{1.88} \mathrm{Sr}_{0.12} \mathrm{CuO}_{4}$, Phys. Rev. B 66, 014524 (2002).

[38] J. Wardh, B. M. Andersen, and M. Granath, Suppression of superfluid stiffness near a Lifshitz-point instability to finitemomentum superconductivity, Phys. Rev. B 98, 224501 (2018).

[39] H.-H. Kim et al., Uniaxial pressure control of competing orders in a high-temperature superconductor, Science 362, 1040 (2018).

[40] R.-H. He, K. Tanaka, S.-K. Mo, T. Sasagawa, M. Fujita, T. Adachi, N. Mannella, K. Yamada, Y. Koike, Z. Hussain, and Z.-X. Shen, Energy gaps in the failed high- $T_{c}$ superconductor $\mathrm{La}_{1.875} \mathrm{Ba}_{0.125} \mathrm{CuO}_{4}$, Nat. Phys. 5, 119 (2009).

[41] E. Razzoli, G. Drachuck, A. Keren, M. Radovic, N. C. Plumb, J. Chang, Y.-B. Huang, H. Ding, J. Mesot, and M. Shi, Evolution from a Nodeless Gap to $d_{x^{2}-y^{2}}$-Wave in Underdoped $\mathrm{La}_{2-x} \mathrm{Sr}_{x} \mathrm{CuO}_{4}$, Phys. Rev. Lett. 110, 047004 (2013). 